\title{
Respective contributions of intestinal Niemann-Pick C1-like 1 and scavenger receptor class B type I to cholesterol and tocopherol uptake: in vivo $v$. in vitro studies
}

\author{
Emmanuelle Reboul $^{1,2,3 *} \dagger$, Zeina Soayfane ${ }^{4,5,6} \dagger$, Aurélie Goncalves ${ }^{1,2,3}$, Michela Cantiello ${ }^{4,5,7}$, \\ Romain Bott $^{1,3}$, Michel Nauze ${ }^{4,5,7}$, François Tercé ${ }^{4,5,7}$, Xavier Collet ${ }^{4,5,7}$ and Christine Coméra ${ }^{4,5,6}$ \\ ${ }^{1}$ INRA, UMR1260 'Lipid Nutrients and Prevention of Metabolic Diseases', Marseille F-13385, France \\ ${ }^{2}$ INSERM, U1025 'Bioavailability of Micronutrients', Marseille F-13385, France \\ ${ }^{3}$ Université Aix-Marseille, Marseille F-13385, France \\ ${ }^{4}$ INSERM, U563, Toulouse F-31024, France \\ ${ }^{5}$ Institut Fédératif de Recherche Bio-Médicale de Toulouse, Université Paul Sabatier, IFR150, Toulouse F-31062, France \\ ${ }^{6}$ TOXALIM, UMR1331-INRA-INP-UPS, Toulouse F-31027, France \\ ${ }^{7}$ INSERM, UMR1048, Toulouse F-31432, France
}

(Submitted 4 March 2011 - Final revision received 9 June 2011 - Accepted 4 July 2011 - First published online 20 September 2011)

\begin{abstract}
The intestinal absorption of cholesterol and lipid micronutrients such as vitamin $\mathrm{E}$ has been shown to share some common pathways. The present study aims to further compare the uptake of cholesterol $\left(\left[{ }^{3} \mathrm{H}\right]\right.$ cholesterol $v$. 22-( $N$-(7-nitrobenz-2-oxa-1,3-diazol-4-yl)amino)-23,24bisnor-5-cholen-3-ol (NBD-cholesterol)) and tocopherol in Caco-2 TC-7 cells and in mouse intestine, with special focus on the respective roles of scavenger receptor class B type I (SR-BI) and Niemann-Pick C1-like 1 (NPC1L1). Conversely to NBD-cholesterol, the uptakes of $\left[{ }^{3} \mathrm{H}\right]$ cholesterol and tocopherol by Caco-2 cells were impaired by both block lipid transport-1 and ezetimibe, which inhibit SR-BI and NPC1L1, respectively. These inhibitions occurred only when cholesterol or tocopherol was delivered to cells included in micelles that contained biliary acid and at least oleic acid as a lipid. In vivo, after $2 \mathrm{~h}$ of digestion in mice, the uptake of the two cholesterol analogues and of tocopherol all showed distinct patterns along the duodenum-jejunum axis. $\left[{ }^{3} \mathrm{H}\right]$ Cholesterol uptake, which correlated closely to NPC1L1 mRNA expression in wild-type (wt) mice, was strongly inhibited by ezetimibe. Intestinal SR-BI overexpression did not change NPC1L1 expression and led to a significant increase in $\left[{ }^{3} \mathrm{H}\right]$ cholesterol uptake in the distal jejunum. Conversely, neither ezetimibe treatment nor SR-BI overexpression had an effect on NBD-cholesterol uptake. However, in contrast with SR-BI mRNA expression, tocopherol absorption increased strongly up to the distal jejunum in wt mice where it was specifically inhibited by ezetimibe, and was increased in the proximal intestine of intestinal SR-BI-overexpressing mice. Thus, cholesterol and tocopherol uptakes share common pathways in cell culture models, but display different in vivo absorption patterns associated with distinct contributions of SR-BI and NPC1L1.
\end{abstract}

Key words: Vitamin E: Cholesterol: Intestinal absorption: Membrane transporters

Over the last few years, there have been significant advances in our understanding of the mechanisms governing the intestinal absorption of dietary lipids and lipid micronutrients. Intestinal cholesterol absorption was initially thought to occur by passive diffusion. It is now well acknowledged that micellar cholesterol uptake by the enterocyte depends mainly on Niemann-Pick C1-like 1 (NPC1L1) activity $^{(1,2)}$, although the molecular mechanisms by which micellar cholesterol crosses the brush-border membrane remain unknown. Other membrane transporters, such as scavenger receptor class B type I (SR-BI), have also been identified, to a lesser extent, as playing roles in cholesterol uptake ${ }^{(3,4)}$ and lipid sensing ${ }^{(5)}$ in the enterocyte.

The roles of NPC1L1 and SR-BI in the intestine are not limited to cholesterol transport. It has recently been shown

Abbreviations: BLT1, block lipid transport 1; DMEM, Dulbecco's minimum essential medium; iSR-BI tg, intestinal scavenger receptor class B type Ioverexpressing transgenic; NBD, 22-(N-(7-nitrobenz-2-oxa-1,3-diazol-4-yl)amino)-23,24-bisnor-5-cholen-3-ol; NPC1L1, Niemann-Pick C1-like 1; SR-BI, scavenger receptor class B type I; wt, wild type.

*Corresponding author: E. Reboul, fax +33 4917821 01, email emmanuelle.reboul@univmed.fr

$\dagger$ These authors contributed equally to the paper. 
that both proteins were involved in the uptake of micellar vita$\min \mathrm{E}^{(6,7)}$, but their relative involvement in tocopherol or cholesterol uptake is not known.

However, note that some of the above observations vary strongly depending on model and experimental conditions used. In particular, in vitro and in vivo data are sometimes conflicting. For example, although it appears clear that SR-BI is involved in cholesterol uptake in brush-border membrane vesicles $^{(8)}$, there are still doubts over its in vivo involvement, as the distribution of the cholesterol absorption along the small intestine in mice fed with a basal diet is not different in $\mathrm{SR}_{-\mathrm{BI}}{ }^{-/}$or wild-type (wt) animals ${ }^{(9)}$. Conversely, although cholesterol uptake in brush-border membrane vesicles prepared from small intestines of wt and NPC1L1 ${ }^{-/-}$mice has been reported as equivalent ${ }^{(8)}$, NPC1L1 visibly has a clear in vivo contribution ${ }^{(10)}$. Indeed, both wt mice treated with ezetimibe and NPC1L1-deficient mice displayed an inhibition from 50 to $70 \%$ of cholesterol absorption. Residual pathways independent of NPC1L1 and ezetimibe are thus responsible for absorption of the remaining $30 \%$ of cholesterol $^{(1)}$ and the totality of fluorescent 22-( $N$-(7-nitrobenz-2-oxa-1,3-diazol-4yl)amino)-23,24-bisnor-5-cholen-3-ol (NBD)-cholesterol ${ }^{(11)}$.

Considering both the similarities and uncertainties related to cholesterol and vitamin E uptake, the aim of the present study was to characterise and compare the uptake of $\left[{ }^{3} \mathrm{H}\right]$ cholesterol, NBD-cholesterol and tocopherol, both in vitro in Caco-2 cells and in vivo in the mouse intestine. Previous studies ${ }^{(12,13)}$ indicated that the transport pathways for cholesterol or vitamin E differ in enterocytes according to distinct micellar delivery. We thus evaluated cholesterol and vitamin $\mathrm{E}$ uptake by comparing the effects of inhibition of SR-BI or NPC1L1 in Caco- 2 cells in combination with three different types of micellar vehicles. These uptake parameters were also analysed in vivo by comparing incorporation along the duodenal-jejunal axis in wt $\mathrm{C} 57 \mathrm{BL} / 6 \mathrm{~J}$ mice against intestinal scavenger receptor class B type I-overexpressing transgenic (iSR-BI tg) mice and wt mice treated with the NPC1L1 inhibitor ezetimibe. We show a clear-cut difference between in vitro and in vivo results, indicating that the human Caco-2 line displays similar pathways for cholesterol and tocopherol uptake, which is not as evident in the mice models. Indeed, in vivo results enabled us to clearly distinguish the respective locations of the intestinal absorption of cholesterol and tocopherol, and to specify where and to what extent NPC1L1 and SR-BI (which are involved in vitro) are physiologically involved during digestion.

\section{Experimental methods}

\section{Chemicals}

$2 R, 4^{\prime} R, 8^{\prime} R$ - $\alpha$-tocopherol ( $\geq 99 \%$ pure) and $2 R, 4^{\prime} R, 8^{\prime} R$ - $\gamma$-tocopherol ( $\geq 97 \%$ pure) were purchased from Flucka (Vaulxen-Velin, France). Tocol, used as internal standard for HPLC analysis, was purchased from Lara Spiral (Couternon, France). $\left[{ }^{3} \mathrm{H}\right]$ Cholesterol was from Amersham (Les Ulis, France). Monoolein, 2-oleoyl-1-palmitoyl-sn-glycero-3-phosphocholine, 1-palmitoyl-sn-glycero-3-phosphocholine, oleic acid, NBD-cholesterol, sodium taurocholate and SDS were purchased from Sigma-Aldrich (Saint-Quentin-Fallavier, France). Block lipid transport 1 (BLT1), a chemical inhibitor of lipid transport mediated by SR-BI, was from Chembridge (San Diego, CA, USA). Ezetimibe, a chemical inhibitor of cholesterol transport mediated by NPC1L1, was purchased from Sequoia Research Products (Reading, UK). Both 1000fold concentrated inhibitor stock solutions were prepared in dimethyl sulphoxide.

\section{Cell culture}

Preparation of micelles. Cholesterol or tocopherol was delivered to the cells in three different types of micelles in Dulbecco's minimum essential medium (DMEM) containing $5 \%$ lipoprotein-deficient serum (prepared as previously described $^{(14)}$ with minor changes) at the final concentrations presented in Table 1. The simplest M1 micelles contained $5 \mathrm{~mm}$ of taurocholic acid in DMEM associated with a mixture of $5 \mu \mathrm{m}$-cholesterol, $7.4 \mathrm{kBq} / \mathrm{ml}\left[{ }^{3} \mathrm{H}\right]$ cholesterol $(1.48 \mathrm{MBq} /$ $\mu \mathrm{mol})$ and $10 \mu \mathrm{m}$-NBD-cholesterol, or $10 \mu \mathrm{m}-\alpha$-tocopherol. The M2 micelles contained oleic acid $(0.5 \mathrm{~mm})$. The M3 micelles also contained oleic acid $(0.5 \mathrm{~mm})$ and other lipids usually found during digestion process. Appropriate volumes of each lipid stock solution were dried under a stream of $\mathrm{N}_{2}$. The lipids were first resuspended in $208 \mu$ l of DMEM containing $24 \mathrm{~mm}$-taurocholate, mixed for $2 \mathrm{~min}$ with a vortex to form the micelles and then diluted to $1 \mathrm{ml}$ in a solution of DMEM and 5\% lipoprotein-deficient serum to reach $5 \mathrm{~mm}$ final of taurocholate $\mathrm{e}^{(15)}$.

\section{Culture conditions}

Caco-2 cell line, clone TC- $7^{(16)}$, was a gift from Dr M. Rousset (UMRS 872, Villejuif, France). Cells were cultured in T-75 flasks in DMEM supplemented with $10 \%$ heat-inactivated fetal bovine serum, $1 \%$ non-essential amino acid and $1 \%$ antibiotics (complete medium), as previously described ${ }^{(17)}$.

\section{Uptake experiments}

Cells were seeded in twenty-four-well plates at a density of 20000 cells per well, and then differentiated for $20-21 \mathrm{~d}$.

Table 1. Lipid composition of the three types of micelles used in cell experiments*

\begin{tabular}{lccc}
\hline Lipids & $\begin{array}{c}\text { Micelles } \\
\text { no. 1 } \\
\text { (M1, mM) }\end{array}$ & $\begin{array}{c}\text { Micelles } \\
\text { no. 2 } \\
\text { (M2, mM) }\end{array}$ & $\begin{array}{c}\text { Micelles } \\
\text { no. 3 } \\
\text { (M3, mM) }\end{array}$ \\
\hline Taurocholate & 5 & 5 & 5 \\
Oleic acid & - & 0.5 & 0.5 \\
$\begin{array}{l}\text { Phosphatidylcholine } \\
\text { Lysophosphatidylcholine }\end{array}$ & - & - & 0.04 \\
Monoolein & - & - & 0.16 \\
\hline
\end{tabular}

Phosphatidylcholine, 2-oleoyl-1-palmitoyl-sn-glycero-3-phosphocholine; lysophosphatidylcholine, 1-palmitoyl-sn-glycero-3-phosphocholine.

* Micelles were prepared as described in Experimental methods. Each type of micelle contained a mixture of $5 \mu \mathrm{m}$-cholesterol, $10 \mu \mathrm{M}$-NBD-cholesterol and $74 \mathrm{kBq}\left[{ }^{3} \mathrm{H}\right]$-cholesterol $(1.48 \mathrm{MBq} / \mu \mathrm{mol}$ of total cholesterol) or $10 \mu \mathrm{M}-\alpha$-tocopherol. 
Cells were pre-incubated for $16 \mathrm{~h}$ in serum-free medium, and then for $1 \mathrm{~h}$ in medium containing $5 \%$ lipoprotein-deficient serum supplemented with dimethyl sulphoxide (control), $10 \mu \mathrm{m}$-BLT1 or $100 \mu \mathrm{m}$-ezetimibe. For lipid absorption measurement, cells were finally incubated for $1 \mathrm{~h}$ with different micelles supplemented or not with the inhibitors. After incubation, the culture medium was harvested, the cells were washed twice in $500 \mu \mathrm{l}$ PBS containing $5 \mathrm{~mm}$-taurocholate, then lysed in $500 \mu \mathrm{l}$ PBS containing $0 \cdot 25 \%$ SDS. Protein concentrations were estimated on aliquots of cell samples using a bicinchoninic acid kit (Pierce, Montluçon, France).

\section{Cholesterol and $2 \mathrm{R}, 4^{\prime} \mathrm{R}, 8^{\prime} \mathrm{R}$ - $\gamma$-tocopherol uptake in mice}

All animal studies were performed in conformity with the Public Health Service Policy on Human Care and Use of Laboratory Animals and in accordance with the local ethics committee of the Toulouse animal facility platform (Anexplo).

\section{Preparation of emulsions}

For cholesterol and tocopherol delivery to mice, emulsions were prepared as described previously ${ }^{(6)}$ with minor changes. Briefly, for each mouse, $100 \mu \mathrm{l}$ of $9 \% \mathrm{NaCl}$ was added to $100 \mu \mathrm{l}$ of maize oil containing a mixture of $30 \mu \mathrm{g}$ of cholesterol, $10 \mathrm{kBq}$ of $\left[{ }^{3} \mathrm{H}\right]$ cholesterol $(0 \cdot 118 \mathrm{MBq} / \mu \mathrm{mol}$ of total cholesterol) and $5 \mu \mathrm{g}$ of NBD-cholesterol, or $5 \mathrm{mg}$ of $\gamma$-tocopherol. Emulsions were formed by bath sonication (Branson 3510; Branson, Danbury, CT, USA) for $15 \mathrm{~min}$ at $0^{\circ} \mathrm{C}$, and were freshly used for force feeding. $\gamma$-Tocopherol was chosen instead of $\alpha$-tocopherol as (i) it shows no discrimination in absorption and (ii) because $\boldsymbol{\gamma}$-tocopherol, in contrast to $\alpha$-tocopherol, is not detectable in the intestine by HPLC before oral administration ${ }^{(6,18)}$.

\section{Animals}

Mice overexpressing SR-BI in the intestine were generated as previously described ${ }^{(4)}$. C57BL/6 Rj (wt) mice were purchased from Janvier (Le-Genest-St-Isle, France). They were housed in a temperature-, humidity- and light-controlled room. They were given a standard chow diet with water ad libitum and were fasted overnight before oral administration. To assess the effect of ezetimibe, mice received two intraperitoneal injections of $10 \mathrm{mg} / \mathrm{kg}$ ezetimibe at 27 and $4 \mathrm{~h}$ before oral administration. At $2 \mathrm{~h}$ after oral administration, the mice were killed and the first $20 \mathrm{~cm}$ of their intestine was harvested downstream of the biliary canal. Intestines were carefully washed with PBS and $5 \mathrm{~mm}$-taurocholate, and cut into four fragments corresponding to the duodenum (first $4 \mathrm{~cm}$ ), the proximal jejunum $(4 \mathrm{~cm})$, the medium jejunum $(6 \mathrm{~cm})$ and the distal part of the jejunum $(6 \mathrm{~cm})$. Samples for tocopherol analysis were flushed with a stream of $\mathrm{N}_{2}$ to prevent oxidation. All samples were stored at $-80^{\circ} \mathrm{C}$ until analysis.

For cholesterol measurement, the fragments were resuspended in $1-1.5 \mathrm{ml}$ PBS, $0.25 \%$ SDS and homogenised using an Ultra-Turrax homogeniser (Labomoderne, Paris, France). Radioactivity was determined by liquid scintillation counting, and the fluorescence of NBD-cholesterol was quantified using a spectrofluorimeter (Varioskan; Thermo Scientific, Courtabouf, France), with excitation and emission wavelengths of 470 and $556 \mathrm{~nm}$, respectively. For tocopherol measurement, samples were resuspended in $500 \mu \mathrm{l}$ PBS and ground with two 3-mm-diameter stainless-steel balls in $2 \mathrm{ml}$ Eppendorf tubes using a MM301 ball mill (Retsch, Eragnysur-Oise, France).

\section{Tocopherol extraction and HPLC analysis}

Tocopherol was extracted from $500 \mu \mathrm{l}$ aqueous samples using tocol as an internal standard, as previously described ${ }^{(6,18)}$. Tocopherol and tocol were then separated using a $250 \times 4.6 \mathrm{~nm} \mathrm{RP} \mathrm{C} \mathrm{C}_{18}, 5 \mu \mathrm{m}$ Zorbax column (Interchim, Montluçon, France) and a guard column. The mobile phase was $100 \%$ methanol. Flow rate was $1.5 \mathrm{ml} / \mathrm{min}$, and the column was kept at a constant temperature $\left(30^{\circ} \mathrm{C}\right)$. The HPLC system comprised a Dionex separation module (P680 HPLC Pump and ASI-100 Automated Sample Injector; Dionex, Aix-en-Provence, France) and a Jasco fluorimetric detector (Jasco, Nantes, France). Tocopherols were detected at $325 \mathrm{~nm}$ after light emission at $292 \mathrm{~nm}$, and identified by retention time compared with pure $(>95 \%)$ standards. Quantification was performed using Chromeleon software (version 6.50 SP4 Build 1000; Dionex, Aix-en-Provence, France) comparing peak area with standard reference curves. All solvents used were of HPLC grade from SDS (Peypin, France).

\section{RNA extraction and quantitative real-time RT-PCR}

Total RNA was isolated from the four different fragments of intestine using the TRIzol method (Invitrogen, Cergy-Pontoise, France). Complementary DNA was reverse-transcribed from $2 \mu \mathrm{g}$ of total RNA and diluted to $20 \mathrm{ng} / \mu \mathrm{l}$ using sterilised water. Quantitative real-time RT-PCR was performed on $12.5 \mathrm{ng}$ of complementary DNA in a final volume of $12.5 \mu \mathrm{l}$, using Power SYBR Green PCR Master Mix and an ABI-Prism 7000 thermal cycler (Applied Biosystems, Villebon-sur-Yvette, France) under standard PCR conditions. Primer sequences are reported in Table 2. All data were normalised to cyclophilin expression levels and were analysed using LinRegPCR ${ }^{(19)}$.

Table 2. Oligonucleotide sequences for quantitative real-time PCR

\begin{tabular}{llll}
\hline Gene & NCBI Refseq & Forward primer $\left(5^{\prime}-3^{\prime}\right)$ & Reverse primer $\left(5^{\prime}-3^{\prime}\right)$ \\
\hline Cyclophilin & NM_011149 & tggagagcaccaagacagaca & tgccggagtcgacaatgat \\
SR-BI & NM_016741 & tctacatcaaatctgtcaagggcat & actggctcgatcttcctgtt \\
NPC1L1 & NM_207242 & gacagatcccaacttgaggt & aaccgtcaggtattgctggtaga
\end{tabular}

SR-BI, scavenger receptor class B type I; NPC1L1, Niemann-Pick C1-like 1. 


\section{Membrane preparation and protein analysis}

The mice were killed and the intestine was removed and cut into four fragments, as described above. Each section was opened and the mucosa was scraped, immediately frozen in liquid $\mathrm{N}_{2}$ and stored at $-80^{\circ} \mathrm{C}$. Tissues were then homogenised at $4^{\circ} \mathrm{C}$ in PBS, as previously described ${ }^{(4)}$. Proteins were determined using a bicinchoninic acid kit, and $50 \mu \mathrm{g}$ were used for western blot analysis. Proteins were separated by SDS-PAGE using $12 \%$ gels under reducing conditions according to Laemmli ${ }^{(20)}$ and were electrophoretically transferred to polyvinylidene fluoride membranes according to Burnette ${ }^{(21)}$. The blotting membrane was incubated with rabbit polyclonal anti-SR-BI IgG (NB400-104; Novus Biologicals, Littleton, CO, USA) or with a 1:500 dilution of rabbit serum against NPC1L1 generously donated by Dr Helen Hobbs. For visualisation, monoclonal anti-rabbit IgG (Sigma) were used as secondary antibodies at 1:5000 dilution.

\section{Statistical analysis}

Differences between more than two groups of unpaired data were tested by the non-parametric Kruskal-Wallis test. The non-parametric Mann-Whitney $U$ test was used as a post boc test when the Kruskal-Wallis test showed significant differences between groups. Differences between only two groups of unpaired data were tested by the Mann-Whitney $U$ test. Values of $P<0.05$ were considered to be significant. All statistical analyses were performed using Statview software version 5.0 (SAS Institute, Cary, NC, USA).

\section{Results}

\section{Effect of micellar composition on $\left.{ }^{3} \mathrm{H}\right]$ - and}

NBD-cholesterol or tocopherol uptake via scavenger receptor class $B$ type I and Niemann-Pick C1-like 1 in Caco-2 cells

We first investigated the role of SR-BI and NPC1L1 on the uptake of cholesterol associated with three different types of micelles (Table 1 ) by Caco-2 cells. When compared to M1 micelles, adding oleic acid to taurocholate (M2 micelles) significantly increased the uptake of both $\left[{ }^{3} \mathrm{H}\right]$ - and NBD-cholesterol (Fig. 1(a) and (b)). More complex micelles (M3) induced a further increase in the uptake of both cholesterol analogs. Inhibition of SR-BI (BLT1, $10 \mu \mathrm{M}$ ) or NPC1L1 (ezetimibe, $100 \mu \mathrm{m})$ had no effect on M1 micelle-mediated uptake or NBD-cholesterol uptake in M2 or M3 micelles, but impaired $\left[{ }^{3} \mathrm{H}\right]$ cholesterol uptake in M2 (-30\% inhibition) and $\mathrm{M} 3$ ( $50 \%$ inhibition) micelles. This indicated that the uptake of $\left[{ }^{3} \mathrm{H}\right]$ cholesterol and not NBD-cholesterol, which was associated with micelles containing at least oleic acid, was partially dependent on SR-BI or NPC1L1. The effects of the inhibitors were not additive, suggesting similar routing pathways for both proteins.

When the experiment was performed with tocopherol (Fig. 1(c)), we again observed a significantly increased (although less pronounced) uptake among M1, M2 and M3 micelles. The inhibition of SR-BI or NPC1L1 did not affect
M1 micelle-mediated uptake, but strongly decreased tocopherol uptake in M2 and very strongly decreased tocopherol uptake in M3 micelles (M2, -27 and $-46 \%$ and M3, -55 to $-77 \%$ for ezetimibe and BLT1, respectively). BLT1 and ezetimibe has no additive effect.

In vivo uptake of $\left[{ }^{3} \mathrm{H}\right]$ - and NBD-cholesterol or tocopherol in wild-type mice and transgenic mice overexpressing scavenger receptor class $B$ type I in the intestine

We investigated in vivo the intestinal absorption of cholesterol and tocopherol in wt $\mathrm{C} 57 \mathrm{BL} / 6 \mathrm{~J}$ mice treated or not with ezetimibe and in a mouse model of intestinal overexpression of SR-BI that we have previously developed ${ }^{(4)}$. We first quantified the expression of SR-BI and NPC1L1 mRNA in four different fragments of mouse intestine (duodenum and proximal, medium and distal jejunum) from wt or iSR-BI tg mice (Fig. 2). SR-BI mRNA and protein expression decreased along the duodenum-jejunum axis in wt mice, as already described $^{(4)}$, but was strongly increased in all intestinal fragments of iSR-BI tg mice (Fig. 2(a)). This mRNA expression was similar to our previous observations on total intestine (25-fold overexpression). In contrast, NPC1L1 mRNA and protein expression slightly increased from duodenum to medium jejunum in wt mice, but were not modified by intestinal SR-BI overexpression (Fig. 2(b)).

To focus on the intestinal absorption process, we then analysed the uptake of $\left[{ }^{3} \mathrm{H}\right]-$, NBD-cholesterol or tocopherol at $2 \mathrm{~h}$ after feeding in wt mice, which is a time long enough to obtain significant uptake values without significant secretion. Indeed, as the half-time of gastric emptying after a liquid meal is about $15 \mathrm{~min}$ in mice ${ }^{(22)}, 2 \mathrm{~h}$ after oral administration were sufficient to allow mixed micelles to form, make contact with both the duodenal and jejunal mucosa and be taken up by enterocytes. $\left[{ }^{3} \mathrm{H}\right]$-Cholesterol displayed an increased uptake from duodenum to medium jejunum, which was well correlated to NPC1L1 mRNA expression (Fig. 3(a)). Conversely, the uptake of NBD-cholesterol was similar in the first three duodenal and jejunal fragments and decreased by $30 \%$ in the distal fragment (Fig. 3(d)). In contrast, the uptake profile of tocopherol strongly increased along the duodenum-distal-jejunum axis (16-fold increase between the first and the last fragment (Fig. 3(g)), partly in agreement with the increased NPC1L1 mRNA expression, but inversely correlated to SR-BI mRNA expression (Fig. 2).

Cross-comparing uptake values between wt and iSR-BI tg mice revealed a significant increase in the incorporation of $\left[{ }^{3} \mathrm{H}\right]$ cholesterol in the distal jejunum of iSR-BI tg mice (Fig. 3(b)), in agreement with the stronger overexpression of SR-BI mRNA in the distal jejunum (150-fold increase) than in the duodenum (16-fold increase). NBD-cholesterol uptake remained unchanged in the iSR-BI tg mice (Fig. 3(e)), whereas tocopherol uptake was significantly increased in the duodenum (3-2-fold) and proximal jejunum (2-4-fold) in iSR-BI tg mice compared to wt mice (Fig. 3(h)), indicating a major difference in the absorption of these lipid molecules related to the intestinal overexpression of SR-BI. 


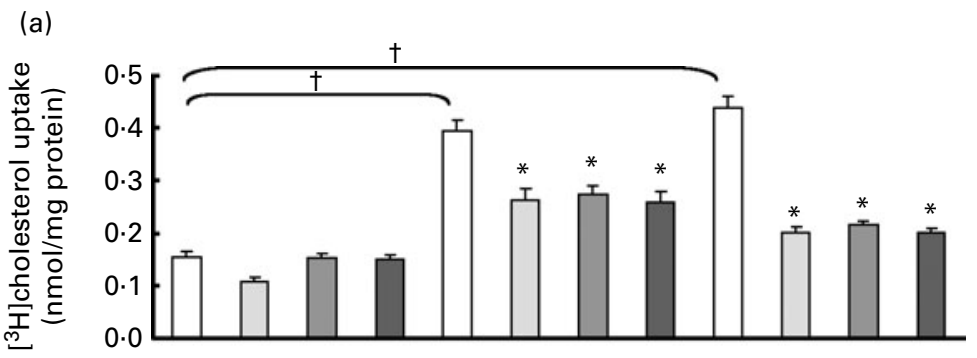

(b)
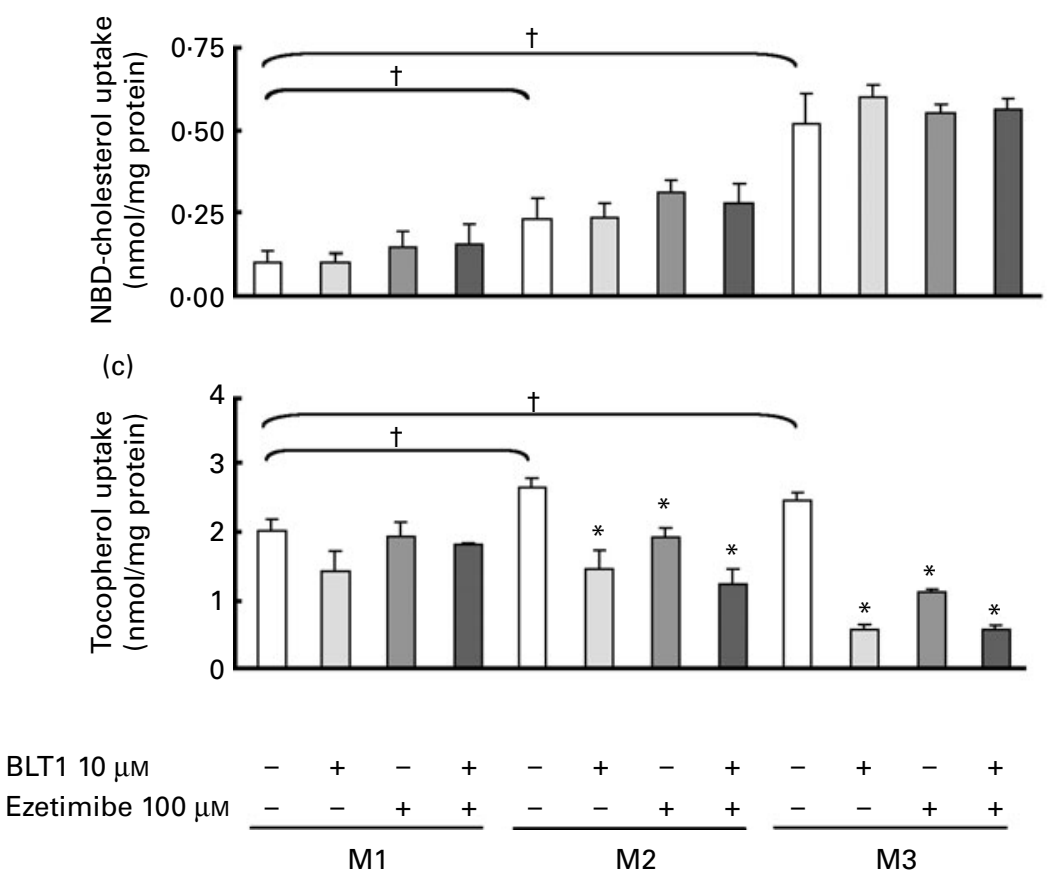

Fig. 1. Effect of micellar lipid composition and transporter inhibitors on $\left[{ }^{3} \mathrm{H}\right]$ cholesterol, 22-( $\mathrm{N}$-(7-nitrobenz-2-oxa-1,3-diazol-4-yl)amino)-23,24-bisnor-5-cholen-3-ol (NBD)-cholesterol and $\alpha$-tocopherol uptake in Caco-2 cells. The combined effects of lipid micellar composition (M1, M2 and M3, see Table 1), scavenger receptor class B type I inhibitor (block lipid transport 1 (BLT1), $10 \mu \mathrm{M}$ ) and Niemann-Pick C1-like 1 inhibitor (ezetimibe; $100 \mu \mathrm{M})$ on (a) [ $\left.{ }^{3} \mathrm{H}\right]$ cholesterol (b) NBD-cholesterol and (c) tocopherol uptake were evaluated in differentiated Caco-2 cells. $\left[{ }^{3} \mathrm{H}\right]$ - and NBD-cholesterol were mixed in each type of micelle (see Experimental methods section). Incubation time was $1 \mathrm{~h}$. Values are means, with their standard errors represented by vertical bars $(n 3) .{ }^{*}$ Mean values were significantly different from those of micelles without inhibitor $(P \leq 0.05)$. † Mean values were significantly different from those of M1 micelles $(P \leq 0.05)$.

Finally, ezetimibe treatment of wt mice strongly decreased the absorption of $\left[{ }^{3} \mathrm{H}\right]$ cholesterol (by over $50 \%$ ) in all four intestinal fragments (Fig. 3(c)). This suggested an active participation of NPC1L1 in $\left[{ }^{3} \mathrm{H}\right]$ cholesterol absorption in the duodenum and jejunum. Ezetimibe also inhibited tocopherol absorption, but only in the distal jejunum, where it is mainly absorbed in control wt mice (Fig. 3(i)). In contrast, NBDcholesterol uptake was not inhibited by ezetimibe, but slightly increased by up to $20 \%$ in duodenum (Fig. 3(f)).

\section{Discussion}

Understanding the molecular mechanisms of lipid absorption by the enterocyte is a critical challenge, as both cholesterol (one-third of total body cholesterol comes from diet) and fat-soluble vitamins may be involved. The objectives of the present study were to compare the absorption of two lipid nutrients, i.e. cholesterol $\left(\left[{ }^{3} \mathrm{H}\right]\right.$ - or NBD-analogues $)$ and tocopherol, by the enterocyte in vitro and in vivo, and to consider the relative involvement of two major membrane lipid transporters, i.e. NPC1L1 and SR-BI, in this process. The Caco-2 cell line is a widely used human intestinal in vitro model giving reproducible results that usually correlate closely with human in vivo figures ${ }^{(23)}$. This cell model has been extensively used to study the absorption of both cholesterol $^{(24-26)}$ and tocopherol ${ }^{(7,27,28)}$. The clone TC-7 was chosen specifically because it is more homogenous than the parent Caco-2 cell line ${ }^{(29)}$ and because it shows good viability after apical incubation with mixed micelles ${ }^{(16)}$. A key step appears to be related to the composition of the lipid donors interacting with uptake efficiency ${ }^{(12,13,15,25,30,31)}$. Here, we used three types of micelles as donor vehicles. The simplest M1 micelles contained only the bile salt taurocholate. More conventional M2 micelles contained, in addition, 10\% oleic acid. Finally, the M3 micelles mimicking the mixed micelles found in dietary conditions ${ }^{(17)}$ were M2 micelles 
(a)

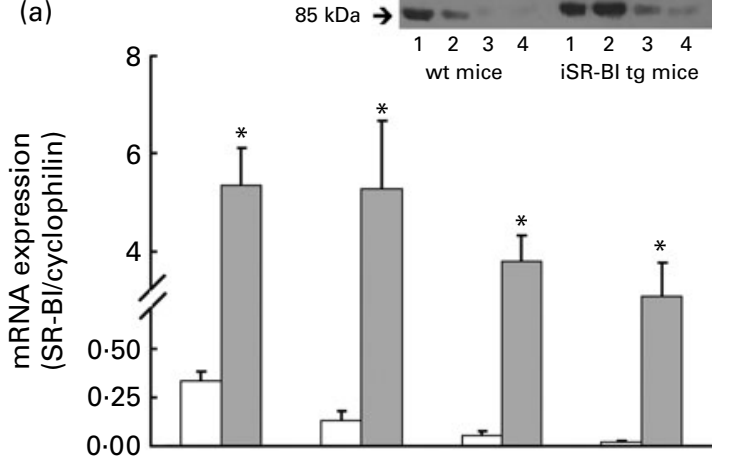

(b)

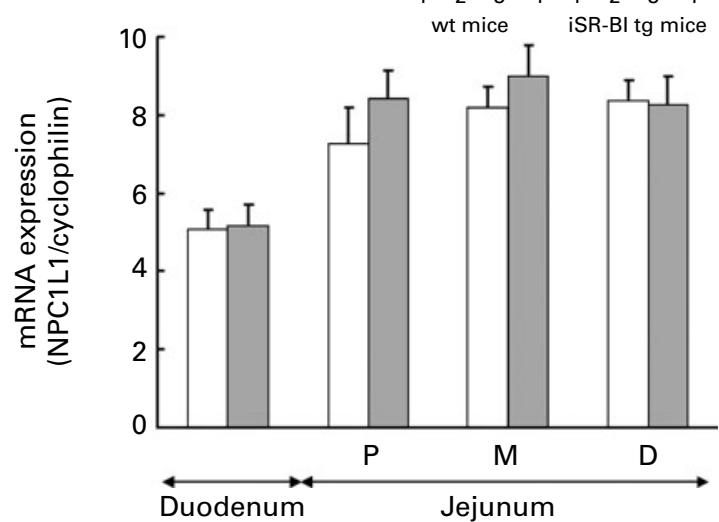

Fig. 2. Scavenger receptor class $B$ type I (SR-BI) and Niemann-Pick C1-like 1 (NPC1L1) expression along the duodenal-jejunal axis in wild-type (wt) and transgenic (tg) mice overexpressing SR-BI in the intestine. Relative expression profiles of (a) SR-BI and (b) NPC1L1 mRNA were analysed by real-time quantitative RT-PCR in wt $(\square)$ and intestinal SR-BI-overexpressing tg mice (iSR-BI $\mathrm{tg}, \square)$ in the duodenum $(4 \mathrm{~cm})$ and in proximal $(P, 4 \mathrm{~cm})$, medium $(\mathrm{M}, 6 \mathrm{~cm})$ and distal $(\mathrm{D}, 6 \mathrm{~cm})$ jejunum fragments. Values were normalised to cyclophilin expression and are expressed as mean values with their standard errors represented by vertical bars, $n 4$ for each group. * Mean values were significantly different from those of wt intestinal fragments $(P \leq 0.05)$. Insets: Western blots of protein expression profiles in different intestinal fragments ((1) duodenum, (2) proximal jejunum, (3) medium jejunum, (4) distal jejunum).

added with three lipids (2-oleoyl-1-palmitoyl-sn-glycero-3phosphocholine, 1-palmitoyl-sn-glycero-3-phosphocholine and monoolein). We clearly demonstrated that specific absorption of $\left[{ }^{3} \mathrm{H}\right]$ cholesterol or tocopherol is mediated by SR-BI or NPC1L1 (between 30 and $70 \%$ inhibition by BLT1 and ezetimibe) when associated with micelles containing at least oleic acid (physiological M3 performing better than M2). Interestingly, we observed a strong effect of micelle complexity on the uptake capacity of $\left[{ }^{3} \mathrm{H}\right]-$ and NBD-cholesterol (more complex micelles were more absorbed, as shown by the 2.5- to 5-fold increase in absorption of M2 and M3 micelles, respectively, compared to M1). However, NBDcholesterol uptake was totally independent of SR-BI or NPC1L1, indicating at least two distinct pathways for the two cholesterol analogs. This could be attributed to structural differences between the two molecules (NBD-cholesterol bears two polar ends, whereas cholesterol is strictly amphiphilic), which could either contribute to a difference of association within the micelles (more at the surface for NBD- cholesterol, more embedded for $\left[{ }^{3} \mathrm{H}\right]$ cholesterol) and/or to differential binding to the hydrophobic extracellular loops found in SR-BI ${ }^{(32)}$ and NPC1L1 ${ }^{(2)}$. These results are consistent with previous data showing that NBD-cholesterol uptake is independent of NPC1L1 and ezetimibe ${ }^{(11)}$, which raises the question of the usefulness of NBD-cholesterol as an analog of cholesterol. In contrast to both cholesterol analogs, tocopherol uptake was less influenced by micellar composition. In this case, the long hydrophobic chain of tocopherol (which is close to a fatty acid structure) may favor an optimal association with the M1 taurocholate micelles.

We found no additive effect of BLT1 and ezetimibe on either $\left[{ }^{3} \mathrm{H}\right]$-cholesterol or tocopherol uptake. Although BLT1 ${ }^{(33,34)}$ and ezetimibe ${ }^{(35)}$ are described as highly specific inhibitors of SR-BI and NPC1L1, respectively, it has also been suggested that they could display a broad target of specificity ${ }^{(36)}$. Indeed, it has already been shown that ezetimibe could block scavenger receptors ${ }^{(25)}$, and that ezetimibe could inhibit cholesterol uptake by brush-border vesicles in the absence of NPC1L1 ${ }^{(8)}$. Our data corroborate with a possible cross-inhibition of SR-BI and NPC1L1. Another possible explanation for the non-additive effect of BLT1 and ezetimibe is that NPC1L1 and SR-BI participate cooperatively in the same lipid transport pathway. Blocking one would then impair the functioning of the other. Interestingly, the knockdown of NPC1L1 in Caco-2 cells has been found to inhibit free cholesterol absorption and decrease SR-BI expression, effectively suggesting cooperation between the two proteins ${ }^{(26)}$

Finally, the effect of oleic acid on the SR-BI- and NPC1L1dependent uptake of cholesterol and tocopherol could also be attributed to a specific increase in chylomicron synthesis, as already observed ${ }^{(10,11)}$. Indeed, cholesterol and tocopherol were recently shown to follow two distinct absorption pathways in vitro in Caco-2 cells ${ }^{(10,11)}$, and also in vivo ${ }^{(12,13,37)}$ with an apoB-dependent routing being specifically stimulated in the presence of oleic acid. The absorption of $\left[{ }^{3} \mathrm{H}\right]$ cholesterol or tocopherol through NPC1L1 or SR-BI observed here may involve this pathway, as absorption is only inhibited in the presence of micelles containing oleic acid. Furthermore, it has recently been shown that oleic acid is able to decrease NPC1L1 expression in Caco-2 cells in conditions similar to our M2 micelles (taurocholate and $0.5 \mathrm{~mm}$-oleate) ${ }^{(38)}$.

In conclusion, this Caco- 2 cell study shows that, in the presence of oleic acid, SR-BI and NPC1L1 seem to be involved in a common absorption pathway that ensures both cholesterol and tocopherol uptake.

In vivo studies were then conducted to pinpoint the respective and physiological contributions of SR-BI and NPC1L1 to cholesterol and tocopherol absorption, using wt mice, iSR-BI tg mice or wt mice treated with ezetimibe. SR-BI mRNA overexpression was found in all four intestinal fragments, as already observed (25-fold increase in the whole intestine), and was correlated to a peak increase in SR-BI protein in the proximal intestine ${ }^{(4)}$. Interestingly, intestinal SR-BI overexpression did not modify NPC1L1 expression, which increased along the duodenum-jejunum axis, as observed in rats ${ }^{(1)}$ and human subjects ${ }^{(26)}$. This differs from the inhibition of SR-BI expression observed in NPC1L1-knockdown Caco-2 cells ${ }^{(31)}$. 

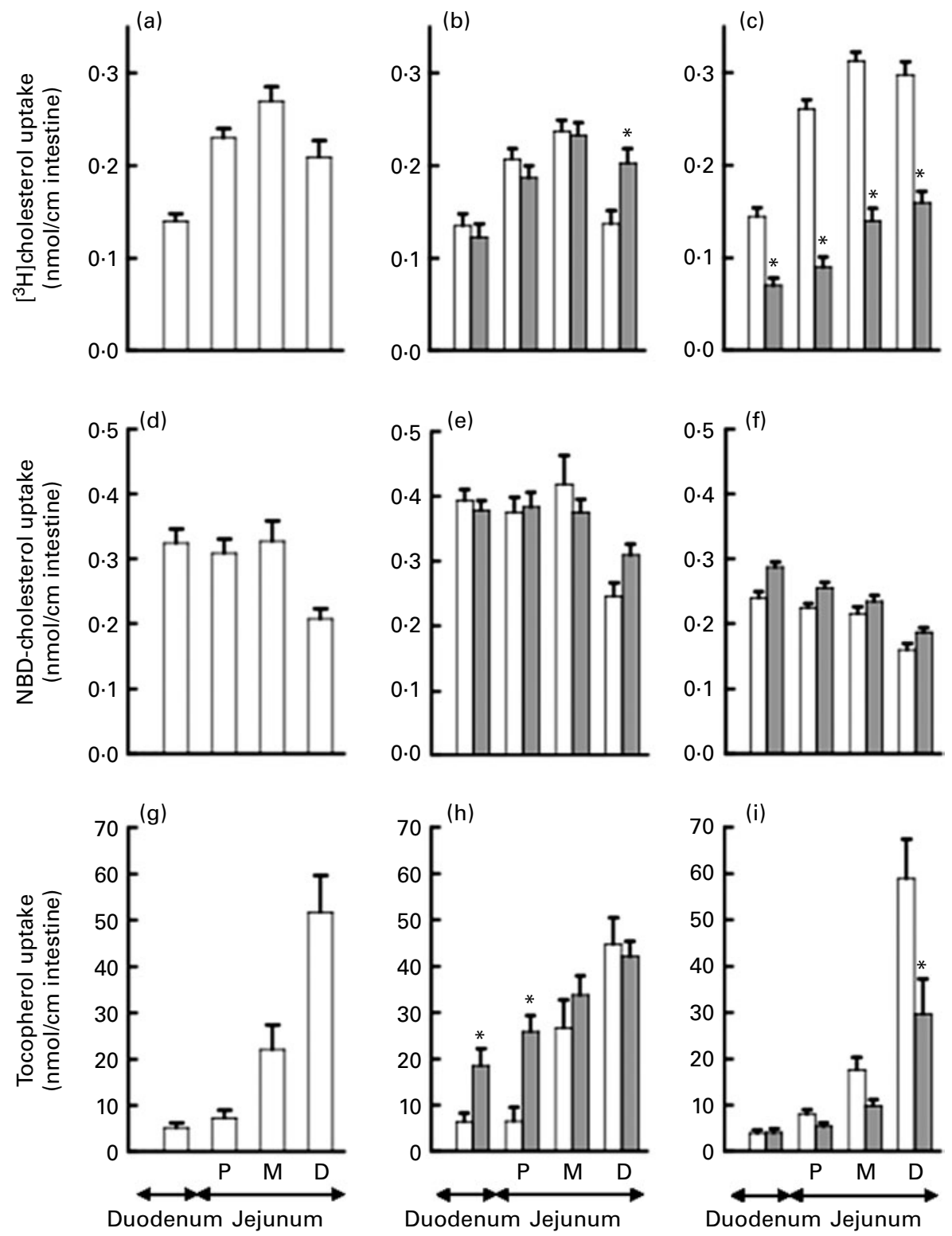

Fig. 3. Comparison of [ $\left.{ }^{3} \mathrm{H}\right]-$, 22-( $N$-(7-nitrobenz-2-oxa-1,3-diazol-4-yl)amino)-23,24-bisnor-5-cholen-3-ol (NBD)-cholesterol and tocopherol uptakes along the duodenal-jejunal axis of mouse intestine in wild-type (wt), scavenger receptor class B type l-overexpressing transgenic (iSR-BI tg) and ezetimibe-treated mice. Mice were force-fed with either $\left[{ }^{3} \mathrm{H}\right]$ - and NBD-cholesterol- or $\gamma$-tocopherol-enriched emulsions. After $2 \mathrm{~h}$, the first $20 \mathrm{~cm}$ of the mouse intestine were harvested downstream of the biliary duct, rinsed and cut into four fragments: the duodenum $(4 \mathrm{~cm})$, and proximal $(P, 4 \mathrm{~cm})$, medium $(M, 6 \mathrm{~cm})$ and distal $(D, 6 \mathrm{~cm})$ jejunum. Results are expressed in $\mathrm{nmol}$ of compound/cm of each intestinal fragment, given as mean values with their standard errors represented by vertical bars. ${ }^{*}$ Mean values were significantly different from those of control (wt) fragment $(P \leq 0.05)$. (a, d, g) wt mice ( $n$ 9); (b, e, h) wt ( $\square) v$. iSR-BI tg mice ( $\square$ ), $n 4-6 / g r o u p ;(c, f$, i) wt mice ( $\square$ ) treated with ezetimibe ( $\square$ ), $n$ 4-6/group. $(a-c)\left[{ }^{3} \mathrm{H}\right]$ Cholesterol; (d-f) NBD-cholesterol and (g-i) $\gamma$-tocopherol.

In contrast to what was observed on Caco- 2 cells, the two cholesterol analogues, especially $\left[{ }^{3} \mathrm{H}\right]$ cholesterol, displayed very distinct uptake profiles compared to tocopherol in wt mice as well as in ezetimibe-treated or iSR-BI tg mice. $\left[{ }^{3} \mathrm{H}\right]$-cholesterol uptake increased progressively along the duodenum-jejunum axis and decreased in the distal jejunum, in correlation with NPC1L1 expression. NBD-cholesterol uptake was similar in the duodenum and the proximal and medium jejunum, and slightly decreased in the distal jejunum fragment. In contrast, tocopherol uptake increased very sharply along the intestinal axis. None of the profiles correlated to SR-BI expression in wt mice. Ezetimibe treatment led to a drastic decrease of $\left[{ }^{3} \mathrm{H}\right]$ cholesterol uptake. These results confirm the major in vivo involvement of NPC1L1 in this process $^{(1)}$. The significant increase in $\left[{ }^{3} \mathrm{H}\right]$ cholesterol uptake, specifically in the distal jejunum in our iSR-BI tg mice, is in agreement with other reports suggesting a moderate collateral role of scavenger receptors in cholesterol absorption ${ }^{(9,39)}$ and with the increased absorption of cholesterol that we had previously observed in this model ${ }^{(4)}$. This increase was actually more pronounced at $4 \mathrm{~h}$ after oral administration, i.e. during digestion and after transport and secretion into the blood ${ }^{(4)}$. 


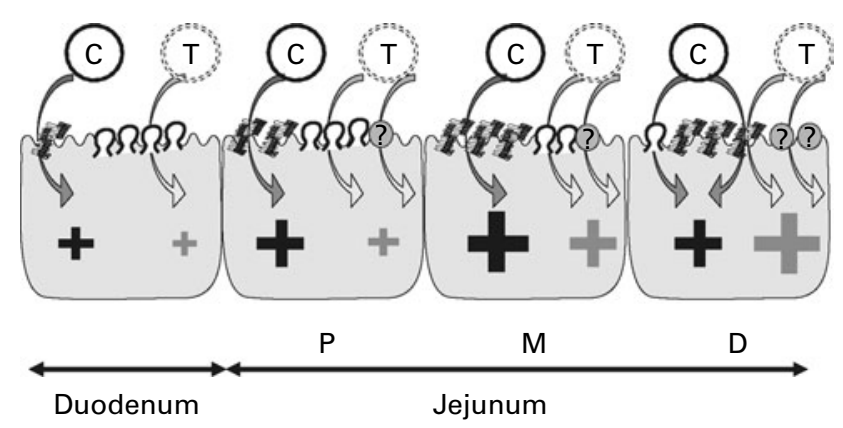

Fig. 4. Uptakes of intestinal cholesterol and vitamin $E$ along the duodenaljejunal axis. Micellar cholesterol (G) and vitamin $E(\mathrm{C})$ are taken up by the enterocyte by Niemann-Pick C1-like 1 (NPC1L1, $\&^{p}$ ), scavenger receptor class B type I (SR-BI, $\Omega$ ) or an unknown transporter (a) in the different intestinal fragments, namely, the duodenum $(4 \mathrm{~cm})$ and proximal $(P, 4 \mathrm{~cm})$, medium $(\mathrm{M}, 6 \mathrm{~cm})$ and distal $(\mathrm{D}, 6 \mathrm{~cm})$ jejunum. Uptake efficiency is symbolised by more or less extended ' + '. Arrows indicate the uptake receptor involved.

Conversely, tocopherol absorption showed a different pattern to $\left[{ }^{3} \mathrm{H}\right]$-cholesterol absorption in different mice models. Ezetimibe treatment had a small effect on vitamin $\mathrm{E}$ absorption in the proximal to medium intestine, but induced a significant $30 \%$ decrease in the distal jejunum compared to wt mice, in agreement with the maximum uptake and the high expression of NPC1L1 observed in this fragment. This confirms that NPC1L1 is also a contributor to in vivo tocopherol absorption. These findings are consistent with a previous study that reported ezetimibe-sensitive absorption of tocopherol by rat intestine $^{(7)}$. The overexpression of SR-BI led to a significant apparent increase in $\gamma$-tocopherol uptake in the proximal part of the intestine, in accordance with our previous data ${ }^{(6)}$. However, when compared to the basal uptake values in wt mice ( 8 and $10 \mu \mathrm{g} / \mathrm{cm}$ for duodenum and proximal jejunum, respectively; Fig. 3(c)), these 3.2- and 2.4-fold increases in iSR-BI tg correspond to 25.6 and $24.4 \mu \mathrm{g} / \mathrm{cm}$ for both fragments, which is much lower than the uptakes observed in wt medium- and distal jejunum $(58$ and $130 \mu \mathrm{g} / \mathrm{cm}$, respectively). These results show for the first time that tocopherol intestinal absorption mainly occurs in the distal part of mouse intestine. SR-BI primarily contributes to vitamin E low-level absorption in the proximal intestine, whereas NPC1L1 principally contributes to its high-level distal absorption. However, the drastic increase in tocopherol absorption found in the medium and distal jejunal fragments strongly suggests a regulated process, almost certainly involving another specific unknown receptor.

Finally, based on the experiments in Caco-2 cells and as expected $^{(11)}$, neither overexpression of SR-BI nor ezetimibe treatment decreased the absorption of NBD-cholesterol, showing that neither SR-BI nor NPC1L1 are implicated in NBDcholesterol uptake. NBD-cholesterol absorption was even stimulated when the ezetimibe-dependent pathway was inhibited. Our results further underline that the chemical modification from cholesterol to NBD-cholesterol has a real impact in apical receptor recognition and uptake by the enterocyte.

These in vivo results, summarised in Fig. 4, refine the data obtained in Caco-2 cells. NBD-cholesterol does not appear to be a suitable cholesterol tracer of the uptake process by
SR-BI or NPC1L1. Moreover, although both NPC1L1 and SR-BI are involved in the uptake of cholesterol and vitamin $\mathrm{E}$ in vitro, NPC1L1 is definitely critical for the absorption of cholesterol in vivo and contributes, although to a lesser extent, to vitamin E uptake. The iSR-BI transgenic model strengthens the argument for a collateral role of SR-BI. The difference observed between Caco- 2 cells and the mouse model could be partly explained by the fact that Caco- 2 cells present a different protein pattern to in vivo enterocytes. In particular, Caco-2 cells highly express both $\mathrm{SR}_{-\mathrm{BI}}{ }^{(17)}$ and NPC1L1 ${ }^{(26)}$, whereas other lipid transporters may not be expressed at all ${ }^{(40)}$. The route of lipid delivery, i.e. artificial $v$. physiological micelles, could also be important, along with the transporter locations along the intestine and inside the enterocytes.

Thus, despite the fact that cholesterol and vitamin E share common uptake pathways through SR-BI and NPC1L1 in vitro, their uptake profiles along the intestine are highly different, suggesting that other mechanisms co-occur during the digestion-absorption process. Further investigations are needed to understand the delayed tocopherol absorption to the distal part of the intestine compared to cholesterol. Finally, the identification of additional intestinal transporters of fat nutrients remains an essential issue.

\section{Acknowledgements}

The present study was funded by PNRA (National program for diet and nutrition research) project no. 5.34 'ABSINTE'. M. C. received financial support under PNRA no. 5.34 'ABSINTE' and Z. S. received a grant from the French Ministry of Research and Higher Education. C. C., E. R. and X. C. conceived the study; E. R., Z. S., A. G., M. C., R. B., M. N. and C. C. conducted the experiments; E. R., Z. S. and C. C. analysed the data. All authors participated in the interpretation of the results. E. R., C. C., F. T. and Z. S. wrote the report. All authors read and approved the final manuscript. The authors are grateful to P. Borel and D. Lairon for helpful discussions. The authors have no conflicts of interest to declare.

\section{References}

1. Altmann SW, Davis HR Jr, Zhu LJ, et al. (2004) Niemann-Pick C1 like 1 protein is critical for intestinal cholesterol absorption. Science 303, 1201-1204.

2. Davis HR Jr \& Altmann SW (2009) Niemann-Pick C1 like 1 (NPC1L1) an intestinal sterol transporter. Biochim Biophys Acta 1791, 679-683.

3. Altmann SW, Davis HR Jr, Yao X, et al. (2002) The identification of intestinal scavenger receptor class B, type I (SR-BI) by expression cloning and its role in cholesterol absorption. Biochim Biophys Acta 1580, 77-93.

4. Bietrix F, Yan D, Nauze M, et al. (2006) Accelerated lipid absorption in mice overexpressing intestinal SR-BI. $J$ Biol Chem 281, 7214-7219.

5. Beaslas O, Cueille C, Delers F, et al. (2009) Sensing of dietary lipids by enterocytes: a new role for SR-BI/CLA-1. PLoS One 4, e4278 
6. Reboul E, Klein A, Bietrix F, et al. (2006) Scavenger receptor class B type I (SR-BI) is involved in vitamin $\mathrm{E}$ transport across the enterocyte. $J$ Biol Chem 281, 4739-4745.

7. Narushima K, Takada T, Yamanashi Y, et al. (2008) Niemannpick C1-like 1 mediates alpha-tocopherol transport. Mol Pharmacol 74, 42-49.

8. Labonte ED, Howles PN, Granholm NA, et al. (2007) Class B type I scavenger receptor is responsible for the high affinity cholesterol binding activity of intestinal brush border membrane vesicles. Biochim Biophys Acta 1771, 1132-1139.

9. Nguyen DV, Drover VA, Knopfel M, et al. (2009) Influence of class B scavenger receptors on cholesterol flux across the brush border membrane and intestinal absorption. J Lipid Res 50, 2235-2244.

10. Davis HR Jr, Basso F, Hoos LM, et al. (2008) Cholesterol homeostasis by the intestine: lessons from Niemann-Pick C1 Like 1 (NPC1L1). Atheroscler Suppl 9, 77-81.

11. Adams MR, Konaniah E, Cash JG, et al. (2011) Use of NBDcholesterol to identify a minor but NPC1L1-independent cholesterol absorption pathway in mouse intestine. Am J Physiol Gastrointest Liver Physiol 300, G164-G169.

12. Iqbal J, Anwar K \& Hussain MM (2003) Multiple, independently regulated pathways of cholesterol transport across the intestinal epithelial cells. J Biol Chem 278, 31610-31620.

13. Anwar K, Kayden HJ \& Hussain MM (2006) Transport of vitamin E by differentiated Caco-2 cells. J Lipid Res $\mathbf{4 7}$, 1261-1273.

14. Hannah VC, Ou J, Luong A, et al. (2001) Unsaturated fatty acids down-regulate srebp isoforms $1 \mathrm{a}$ and $1 \mathrm{c}$ by two mechanisms in HEK-293 cells. J Biol Chem 276, 4365-4372.

15. Chateau D, Pauquai T, Delers F, et al. (2005) Lipid micelles stimulate the secretion of triglyceride-enriched apolipoprotein B48-containing lipoproteins by Caco-2 cells. J Cell Physiol 202, 767-776.

16. Salvini S, Charbonnier M, Defoort C, et al. (2002) Functional characterization of three clones of the human intestinal Caco-2 cell line for dietary lipid processing. Br J Nutr $\mathbf{8 7}$, 211-217.

17. Reboul E, Abou L, Mikail C, et al. (2005) Lutein transport by Caco- 2 TC-7 cells occurs partly by a facilitated process involving the scavenger receptor class B type I (SR-BI). Biochem J $\mathbf{3 8 7}, 455-461$

18. Reboul E, Trompier D, Moussa M, et al. (2009) ATP-binding cassette transporter A1 is significantly involved in the intestinal absorption of alpha- and gamma-tocopherol but not in that of retinyl palmitate in mice. Am J Clin Nutr 89, 177-184.

19. Ruijter JM, Ramakers C, Hoogaars WM, et al. (2009) Amplification efficiency: linking baseline and bias in the analysis of quantitative PCR data. Nucleic Acids Res 37, e45.

20. Laemmli UK (1970) Cleavage of structural proteins during the assembly of the head of bacteriophage T4. Nature $\mathbf{2 2 7}$, 680-685.

21. Burnette WN (1981) 'Western blotting': electrophoretic transfer of proteins from sodium dodecyl sulfate-polyacrylamide gels to unmodified nitrocellulose and radiographic detection with antibody and radioiodinated protein A. Anal Biochem 112, 195-203

22. Bennink RJ, De Jonge WJ, Symonds EL, et al. (2003) Validation of gastric-emptying scintigraphy of solids and liquids in mice using dedicated animal pinhole scintigraphy. $J \mathrm{NuCl}$ Med 44, 1099-1104.

23. Artursson P, Karlsson J, Ocklind G, et al. (1996) Studying transport processes in absorptive epithelia. In Epithelial
Cell Culture: A Practical Approach, pp. 111-133 [AJ Shaw, editor]. Oxford: IRL Press.

24. Cai L, Eckhardt ER, Shi W, et al. (2004) Scavenger receptor class B type I reduces cholesterol absorption in cultured enterocyte CaCo-2 cells. J Lipid Res 45, 253-262.

25. Werder M, Han CH, Wehrli E, et al. (2001) Role of scavenger receptors SR-BI and CD36 in selective sterol uptake in the small intestine. Biochemistry 40, 11643-11650.

26. Sane AT, Sinnett D, Delvin E, et al. (2006) Localization and role of NPC1L1 in cholesterol absorption in human intestine. J Lipid Res 47, 2112-2120.

27. Traber MG, Goldberg I, Davidson E, et al. (1990) Vitamin E uptake by human intestinal cells during lipolysis in vitro. Gastroenterology 98, 96-103.

28. Kiyose C, Muramatsu R, Fujiyama-Fujiwara Y, et al. (1995) Biodiscrimination of alpha-tocopherol stereoisomers during intestinal absorption. Lipids 30, 1015-1018.

29. Gres MC, Julian B, Bourrie M, et al. (1998) Correlation between oral drug absorption in humans and apparent drug permeability in TC-7 cells, a human epithelial intestinal cell line: comparison with the parental Caco-2 cell line. Pharm Res 15, 726-733.

30. Haikal Z, Play B, Landrier JF, et al. (2008) NPC1L1 and SR-BI are involved in intestinal cholesterol absorption from smallsize lipid donors. Lipids $\mathbf{4 3}, 401-408$.

31. Landrier JF, Malezet-Desmoulins C, Reboul E, et al. (2008) Comparison of different vehicles to study the effect of tocopherols on gene expression in intestinal cells. Free Radic Res 42, 523-530.

32. Gu X, Trigatti B, Xu S, et al. (1998) The efficient cellular uptake of high density lipoprotein lipids via scavenger receptor class B type I requires not only receptor-mediated surface binding but also receptor-specific lipid transfer mediated by its extracellular domain. J Biol Chem 273, 26338-26348.

33. Nieland TJ, Penman M, Dori L, et al. (2002) Discovery of chemical inhibitors of the selective transfer of lipids mediated by the HDL receptor SR-BI. Proc Natl Acad Sci U $S$ A 99, 15422-15427.

34. Nieland TJ, Shaw JT, Jaipuri FA, et al. (2008) Identification of the molecular target of small molecule inhibitors of HDL receptor SR-BI activity. Biochemistry 47, 460-472.

35. Garcia-Calvo M, Lisnock J, Bull HG, et al. (2005) The target of ezetimibe is Niemann-Pick C1-like 1 (NPC1L1). Proc Natl Acad Sci U S A 102, 8132-8137.

36. Nieland TJ, Chroni A, Fitzgerald ML, et al. (2004) Cross-inhibition of SR-BI- and ABCA1-mediated cholesterol transport by the small molecules BLT-4 and glyburide. J Lipid Res 45, 1256-1265.

37. Iqbal J \& Hussain MM (2005) Evidence for multiple complementary pathways for efficient cholesterol absorption in mice. J Lipid Res 46, 1491-1501.

38. Chen J, Li Q, Zhang Y, et al. (2010) Oleic acid decreases the expression of a cholesterol transport-related protein (NPC1L1) by the induction of endoplasmic reticulum stress in CaCo-2 cells. J Physiol Biochem 67, 153-163.

39. van Bennekum A, Werder M, Thuahnai ST, et al. (2005) Class B scavenger receptor-mediated intestinal absorption of dietary beta-carotene and cholesterol. Biochemistry $\mathbf{4 4}$, 4517-4525.

40. Nassir F, Wilson B, Han X, et al. (2007) CD36 is important for fatty acid and cholesterol uptake by the proximal but not distal intestine. J Biol Chem 282, 19493-19501. 